\title{
Talbot bands and temporal processing of optical signals
}

J. Jahns

jahns@fernuni-hagen.de

A.W. Lohmann

M. Bohling
FernUniversität Hagen, Optische Nachrichtechnik, Universtitätsstr. 27/PRG, 58084 Hagen, Germany

Universität Erlangen-Nürnberg, Lehrstuhl für Multimediakommunikation und Signalverarbeitung, Cauerstr. 7, 91058 Erlangen, Germany

FernUniversität Hagen, Optische Nachrichtechnik, Universtitätsstr. 27/PRG, 58084 Hagen, Germany

Talbot's bands can be observed in one of the diffraction orders of a grating spectrometer with a partially phase-shifted input beam. This experiment has long been considered as a curiosity. Here, we look at it from a new perspective: we discuss the Talbot band experiment as a combination of two kinds of dispersion, refractive and diffractive. This may be of interest for various tasks of temporal optical signal processing such as dispersion management and the shaping of ps/fs-pulses. For this purpose, we demonstrate the use of micro machined optical elements to alter the performance of the classical Talbot band setup. A theoretical description and experimental results are presented. [DOI: 10.2971/jeos.2006.06001]

Keywords: Diffraction and gratings, diffractive optics, Fourier optics and optical signal processing, signal processing, ultrafast optics, pulse shaping, micro-optics

\section{I n t rod u c t io n}

H. F. Talbot is widely recognised for his work on the selfimaging effect published in 1836 [1]. On the other hand, another experiment described by Talbot in 1837 [2] on the formation of interference fringes in white light has gained relatively little attention so far. In that experiment, Talbot observed the formation of interference fringes ("Talbot bands") in one of the two first diffraction orders of a grating spectrometer upon inserting a piece of glass of appropriate thickness halfway into the illuminating beam Figure 1. This observation is somewhat surprising, in particular, the fact that the bands occur only in the +1 st order (notation as defined in the figure) and not in the -1st order. Fringes are shown in Figure 1.

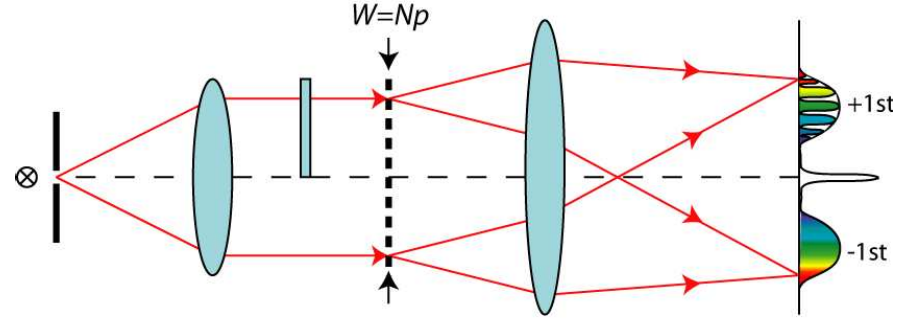
$\mathrm{S}$ GP G O

FIG. 1 Classical experiment for the observation of the Talbot bands ("experiment 1"). Setup: a diffraction grating $G$ is illuminated by a collimated beam of white light from source $S$. A glass plate GP is inserted half-way into the illuminating beam. In the output plane 0 of the grating spectrometer bands are observed only in the +1 st order.

FIG. 2 Observed fringe pattern. Fringe contrast and spacing vary with the wavelength. The reduced brightness between red and green is due to the colour characteristic of the CCD camera. Here, $d=100 \mu \mathrm{m}$ and $p=20 \mu \mathrm{m}$.
The occurrence of the intereference fringes in the +1 st order can be explained qualitatively in the temporal domain. For this, it is suitable to view broadband light as consisting of short pulses. For our brief review of the experiment, we will follow the description presented by Lohmann [3], however, we would like to mention two other articles by Parker Givens [4] and by Benkö, Hilbert and Bor [5].

We assume that the aperture diameter of the setup shown in Figures 1 and 2 is $W=N p$ where $p$ is the period of the grating and $N$ is the number of periods in the grating. Furthermore, we assume that the glass plate covers half of the aperture. Intuitively, it is clear that the experimental observation described in Section 1, Figures 1 and 2, is caused by the asymmetry between the optical paths in the +1 st and the -1 st order. To understand this, we look at three snapshots taken at three different times $t_{1}, t_{2}$ and $t_{3}$ and consider two pulses: one travelling through the upper half of the aperture, i.e., through the glass plate, one travelling through the lower half Figure 3. At $t=t_{1}$, the two pulses are located at the same longitudinal position just before the glass plate. A short time later, at $t=t_{2}$ the upper pulse has passed through the glass plate. It now trails the beam in the lower half which has travelled through air. The optical path length difference is $\Delta n l$. Here, $\Delta n=n-1$ is the index difference between glass and air as a function of the temporal frequency, $d$ is the thickness of the glass plate. We denote the temporal delay between the lower and the upper beam as

$$
t_{r}=\frac{D n d}{c}
$$




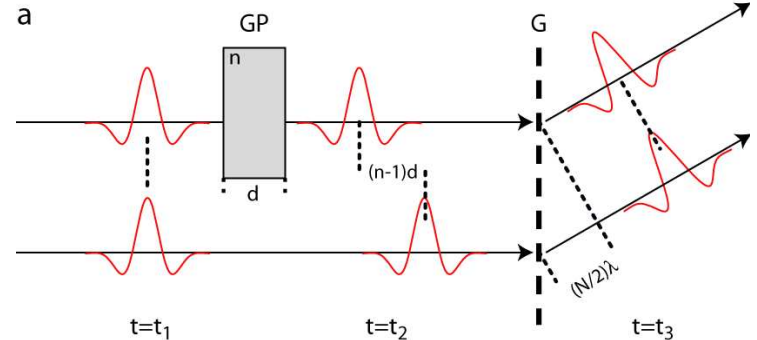

b

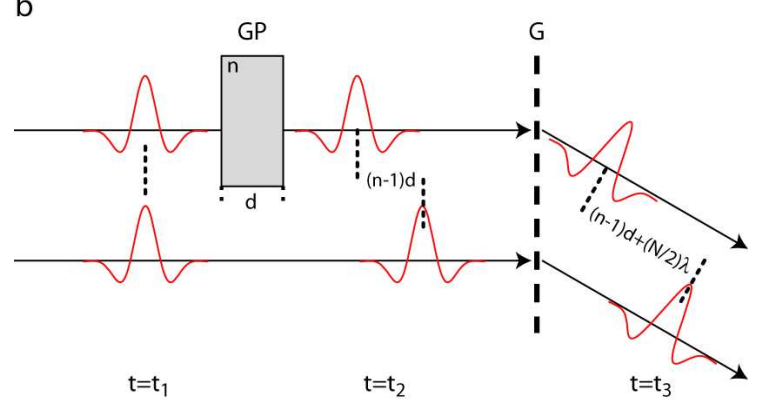

FIG. 3 Three "snapshots" taken at different times for $a$. the +1 st order and $b$. the -1 st order. At $t=t_{1}$ both incoming pulses are at the same z-position. At $t=t_{2}$, the lower pulse is delayed relative to the first by a distance $(n-1) d$, where $d$ is the thickness of the glass plate. $t=t_{3}$ : for $a$. the detour due to grating diffraction is such that the lower pulse is delayed relative to the lower pulse so that both pulses are (approximately) in phase again. In the -1st order $b$. the detour leads to a further delay of the upper pulse relative to the lower pulse, so that both do not overlap. (Remark: The dimensions are not at scale.)

Here, the index ' $r$ ' was chosen to indicate the refractive nature of the delay due to the glass plate. Behind grating $G$, the pulses experience a further time delay, now due to the optical detour caused by diffraction. This delay, denoted $t_{d}$ varies with the diffraction order. In the +1 st order, the pulse in the lower half is delayed with respect to the pulse in the upper half. If we assume a lateral offset between the two pulses of $(N / 2) p$ the detour is $(N / 2) \lambda$. Taking into account the diffraction order $m$, we can write for the delay due to diffraction:

$$
t_{d}=-m\left(\frac{N}{2}\right) \frac{\lambda}{c}
$$

The minus sign reflects the fact that in the +1 st order the time delays between lower and upper pulse have opposite signs. The total time delay between the lower and the upper pulse is:

$$
t=t_{r}+t_{d}
$$

For $m=1$, we obtain:

$$
\Delta n d=(N / 2) \frac{\lambda}{c}
$$

Hence, the delay due to the glass plate and the delay due to the grating diffraction compensate for the central wavelength if the thickness is

$$
d=\frac{N \lambda}{2 \Delta n}
$$

For example, with $\lambda=500 \mathrm{~nm}, N=100$ and $\Delta n \approx 0.5$, one obtains $d \approx 50 \mu \mathrm{m}$.

In the -1 st order, the delay between upper and lower pulse is negative, i.e. the spatial distance between the two pulses is further increased. It is obvious, that the contrast of the interference fringes depends on the time delays. For optimum contrast, the delay between the upper and the lower pulse should be zero which leads to the condition expressed by Eq. (4). It may be noted at this point, that the experiment setup yields good contrast only if the incoming wave is well collimated. A deviation from a plane wavefront results in a temporal blurring across the aperture and therefore also in a noticeable reduction of the fringe contrast.

As explained in the previous section, the difference between the +1 st and the -1 st order can be understood from the differences of the optical paths. Instead of considering the temporal signal $S(t)$, however, we may also view this experiment as processing the signal spectrum $\tilde{S}\left(v_{t}\right)$. This attitude emphasises the quite different dispersion impact of re-fraction and diffraction. By comparison: we are used to understanding light in spectroscopy by the power spectrum $\left|\tilde{S}\left(v_{t}\right)\right|^{2}$. This view does not suffice for understanding the Talbot bands. Here, it is the phase of the signal spectrum that is manipulated.

This remainder of this article is organised as follows: after the review of the Talbot band experiment in this section, we will present a theory to describe the effect in terms of diffraction and refraction (Section 2). A detailed mathematical analysis will follow in Section 3. In Section 4, we will show two demonstration experiments that constitute an extension of the classical Talbot band experiment. In our conclusion, we will discuss particularly the potential use of the Talbot band experiment for temporal processing of ultrashort optical pulses.

\section{TALBOT BANDS AS A RE- FRACTIVE-DIFFRACTIVE PHE- NOM E N O N}

White light described by its temporal field $S(t)$ may be considered to consist of short pulses, a single pulse denoted by $S_{0}(t)$. The spectrum $\tilde{S}(v)$ is given as its Fourier transform with $v$ as the temporal frequency coordinate:

$$
\tilde{S}(v)=\int S(t) \exp (2 \pi i v t) d t
$$

Spectral width $\Delta v$ and pulse duration $\Delta t$ are related. As an example, we consider a Gaussian pulse, denoted as $S_{G}(t)$ :

$$
S_{G}(t)=\exp \left[-(t / \Delta t)^{2}\right] \exp \left(-2 \pi i v_{0} t\right)
$$

with a Fourier spectrum

$$
\tilde{S}_{G}(v)=\sqrt{\pi} \Delta t \exp \left[-\left(\pi \Delta t\left(v-v_{0}\right)\right)^{2}\right]
$$

and a time-bandwidth product:

$$
\Delta t \Delta v=1 / \pi
$$

where $\Delta t$ and $\Delta v$ are the $1 / e$-widths of the signal and the spectrum, respectively. With the relation $|\Delta v|=c|\Delta \lambda| / \lambda_{0}^{2}$, a spectral width $\Delta \lambda \approx 50 \mathrm{~nm}$ around a centre wavelength $\lambda_{0}=550$ $\mathrm{nm}$ corresponds to pulse duration $\Delta t \approx 6 \mathrm{fs}$. Such short pulses were first demonstrated in [6] and pulses of typically less than $30 \mathrm{fs}$ are nowadays generated by commercial laser systems. 
When a pulse passes through an optical element (a phase plate or a grating) or a whole system, it experiences dispersion, i.e., the occurring time delay varies with frequency. In the case of linear devices we can use the concept of the temporal transfer function, here denoted by $\tilde{H}(v)$ to establish the relationship between input and output spectra:

$$
\tilde{S}_{\text {out }}(v)=\tilde{S}_{\text {in }}(v) \tilde{H}(v)
$$

Assuming no absorption and neglecting polarisation effects, we can write for the transfer function $\tilde{H}(v)$ :

$$
\tilde{H}(v)=\exp [2 \pi i t(v) v]
$$

In the qualitative explanation of Section 1, the time delay was considered to be constant. In order to take dispersion into account, we write for the refractive time delay due to the phase plate, $t_{r}(v)$, as a function of the frequency:

$$
t_{r}(v)=\frac{\Delta n(v) d}{c}
$$

For a glass plate with normal (anomalous) dispersion, the index of refraction increases (decreases) with $v$, i.e., $d n / d v>0$ $(d n / d v<0)$ and hence

$$
d t_{r}(v) / d v\left\{\begin{array}{cc}
<0 & \text { anomalous } \\
>0 & \text { normal }
\end{array} .\right.
$$

And for the time delay $t_{d}(v)$ due the grating it is

$$
t_{d}(v)=-m\left(\frac{N}{2}\right) \frac{c}{v}
$$

from which we obtain:

$$
\frac{d t_{d}(v)}{d v}=m\left(\frac{N}{2}\right) \frac{c}{v^{2}}
$$

Notice, that for complementary orders, for example, the +1 st and -1st order, we have opposite dispersive behaviour:

$$
\left.\frac{d t_{d}(v)}{d v}\right|_{m=1}=-\left.\frac{d t_{d}(v)}{d v}\right|_{m=-1}
$$

Although, of course, dispersion is due to diffraction in both cases, one may use the combination of two complementary orders for compensation purposes. This is widely done for achromatization of all-diffractive systems [7] and for pulse shaping devices using double diffraction [8,9]. The point of view of considering the Talbot bands as a combination of refraction and diffraction was discussed in detail in Ref. [3]. In particular, various cases of combining different element have been discussed. We now want to return to the case of the Talbot bands and see how the combination of different refractive and diffractive dispersion characteristics can be exploited. In the next section, we calculate the intensity of the Talbot bands.

\section{W A V E-OP TICAL A N A L Y S I S}

We calculate the far-field pattern dependent on the observation angle $\alpha$. The temporal field travelling along that direction consists of a sequence of pulses (see Figure 4):

$$
S(t, \alpha)=\sum_{m=0}^{N / 2-1} S_{0}(t-m t)+\sum_{m=N / 2}^{N} S_{0}\left(t-m t+t_{r}\right)
$$

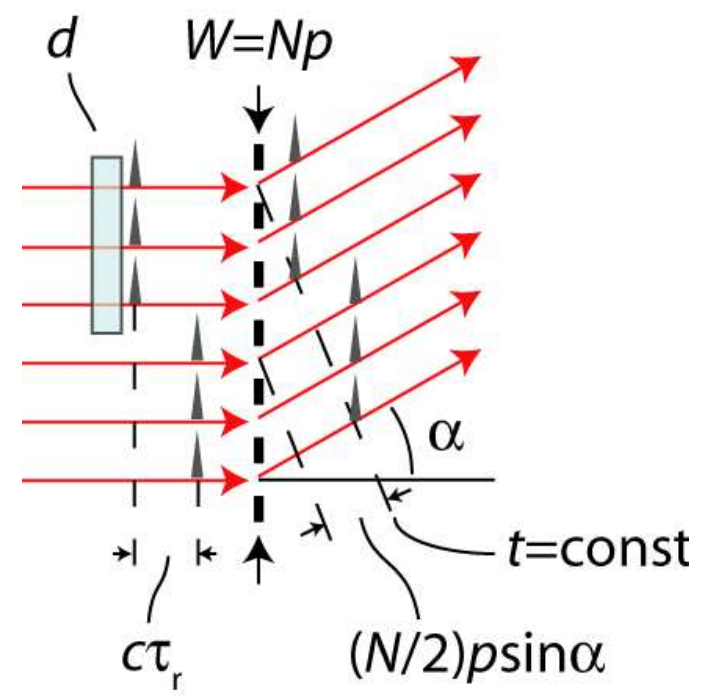

FIG. 4 Explanation of the theoretical calculation for the classical Talbot band experiment. Due to the opposite signs of the refractive delay, $\tau_{r}$, and the diffractive delay, $\tau_{d}=(N / 2) p \sin \alpha / c$, corresponding pulses lie approximately on the same line $t=$ const behind the grating.

Here, $S_{0}(t)$ denotes an individual pulse, $t=\lambda / c$ is the delay between neighbouring slits of the diffraction grating. Fringes can be observed if $t_{r} \approx(N / 2) p \sin \alpha / c$. In the far-field, we observe the intensity $I(\alpha)$. We can write:

$$
\begin{gathered}
I(\alpha)=\int_{(t)}|S(t, \alpha)|^{2} d t=\int_{(v)}|\tilde{S}(v, \alpha)|^{2} d v \\
=\int_{(v)}\left|\tilde{S}_{0}(v)\right|^{2}|\tilde{H}(v, \alpha)|^{2} d v
\end{gathered}
$$

$\tilde{S}_{0}(v)$ is the spectrum of the individual pulse according to Eq. (6). $\tilde{H}(v, \alpha)$ is the transfer function of the optical setup along the direction of observation. The left side of this equation states that the intensity is given by integrating over the intensities in the pulse sequence. The right side follows from Eq. (10) and Parseval's theorem. We can further split up the transfer function into two terms:

$$
\begin{gathered}
\tilde{H}(\nu, \alpha)=\sum_{m=0}^{N / 2-1} \exp (2 \pi i v m t)+ \\
\sum_{m=0}^{N / 2-1} \exp \left[2 \pi i v\left(\left(m+\frac{N}{2}\right) t-t_{r}\right)\right] \\
=\left\{1+\exp \left[2 \pi i v\left(N t / 2-t_{r}\right)\right]\right\} \cdot \sum_{m=0}^{N / 2-1} \exp (2 \pi i v m t) \\
=\tilde{F}(\nu, \alpha) \cdot \tilde{G}(\nu, \alpha)
\end{gathered}
$$

and obtain the following expression for the intensity (by dropping a factor of 2):

$$
I(\alpha)=\int\left|\tilde{S}_{0}(v)\right|^{2}|\tilde{F}(\nu, \alpha)|^{2}|\tilde{G}(v, \alpha)|^{2} d v
$$

The first term in the integral, $\left|\tilde{S}_{0}(v)\right|^{2}$ is the power spectrum of the optical pulse. The third term, 


$$
|\tilde{G}(v)|^{2}=\left|\sum_{m=0}^{N / 2-1} \exp (2 \pi i m v t)\right|^{2}=\frac{\sin ^{2}(\pi(N / 2) v t)}{\sin ^{2}(\pi v t)},
$$

is the typical term that one obtains for multiple beam interference and describes the different diffraction orders. The modulation of the diffraction orders, i.e., the structure of the Talbot bands, is given by the second term, $|\tilde{F}(v)|^{2}$. For the classical Talbot band experiment, one obtains:

$$
|\tilde{F}(v)|^{2}=2[1+\cos (2 \pi \phi)]
$$

with

$$
\phi=v\left(\frac{N}{2} t-t_{r}\right)=\frac{N}{2} v p \sin \alpha / c-\Delta n d v / c
$$

The sinusoidal modulation is typical for two-beam interferometers. Its occurrence is not surprising here, since the incoming beam is subdivided into two beamlets by the glass plate. Since both share the same aperture, we may call the conventional Talbot band setup an "in-line two-beam interferometer." This terminology is introduced here, since later we want to extend the experiment to a multi-beam setup. The phase term $\phi$ consists of two terms. The first term varies with the observation angle $\alpha$ :

$$
\frac{d \phi}{d \alpha}=\frac{N}{2} \cdot \frac{v}{c} p \cos \alpha
$$

The inverse term, $1 /(d \phi / d \alpha)$ is the period of the fringes, here denoted as $p_{T}$ :

$$
p_{T}=\frac{c / v}{(N / 2) p \cos \alpha}=\frac{\lambda}{(W / 2) \cos \alpha}
$$

with $W=N p$ the beam aperture. For small angles we can approximate $p_{T} \approx 2 \lambda / W$. Obviously the modulation period of the Talbot bands increases the wavelength as confirmed by Figure 3. The phase term $\phi$ contains a second term, $\Delta n d v / c$. This term is independent of $\alpha$, however, it also varies with the frequency or the wavelength, respectively. The fringe contrast is highest where the cosine function is near the value 1, i.e., for $\phi \approx 0$ and hence around the angle

$$
\sin \alpha \approx \frac{\Delta n d}{W / 2}
$$

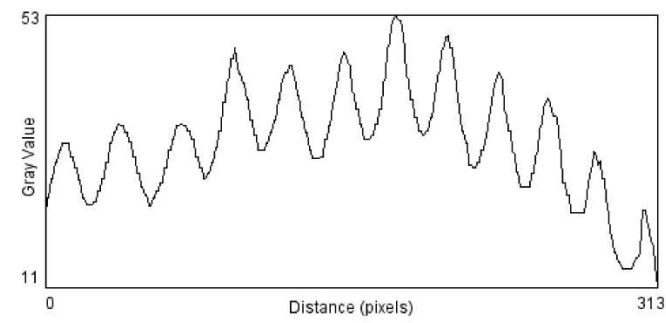

FIG. 5 Measured intensity distribution in the +1 st order of Figure 2.

\section{EXTENSION O F THE TALBOT B A N D EXPERIMET}

The mathematical description of the previous section suggests to modify the classical Talbot band experiment so that more than two interfering beams are generated. Here we discuss the extension of the experiment in different ways. If, instead of a simple glass plate a structured phase element is inserted into the aperture, we can achieve a more complex temporal modulation across the incoming beam. We demonstrate this for two cases denoted as experiments 2 and 3 .

First, we generalise our theory. We assume that instead of a simple glass plate, a structured phase element is used. Suppose it consists of $K$ discrete steps of equal width $w=M p$, i.e., . $M K=N$. Then we can re-write Eq. (17) as

$$
S(t, \alpha)=\sum_{k=0}^{K-1} \sum_{m=0}^{M-1} S_{0}\left[t-(m+k M) t+t_{r}^{(k)}\right]
$$

$t_{r}^{(k)}$ is the time delay generated by the k-th interval. For the classical Talbot band experiment, it is $K=2, M=N / 2$ and $t_{r}^{(k)}=k t_{r}$. With Eq. (17), we can easily find:

$$
\begin{gathered}
\tilde{H}(\nu, \alpha)=\sum_{k=0}^{K-1} \sum_{m=0}^{M-1} \exp \left[2 \pi i v\left((m+k M) t-t_{r}^{(k)}\right)\right] \\
=\sum_{k=0}^{K-1} \exp \left[2 \pi i v\left(k M t-t_{r}^{(k)}\right)\right] \cdot \sum_{m=0}^{M-1} \exp (2 \pi i m v t) \\
=\tilde{F}(v, \alpha) \cdot \tilde{G}(\nu, \alpha)
\end{gathered}
$$

In experiment 2, the phase object consisted of a linear staircase as shown in Figure 6. Width and height of the steps have to be chosen appropriately: since the aperture is subdivided into more and narrower steps, the step height has to be reduced. The condition is derived from $\Delta n d^{\prime}=w \sin \alpha$ from which follows (analog to Eq. (4)): 


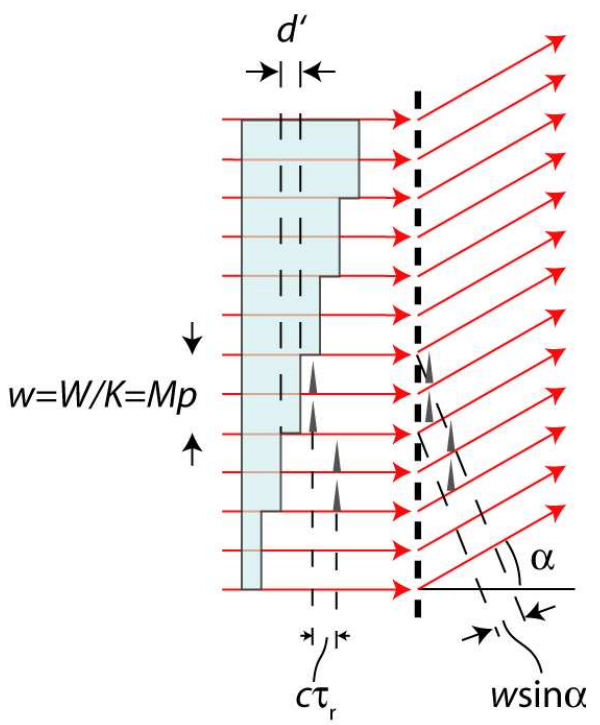

FIC. 6 Experiment 2. Calculation of the step height and width for a staircase object.

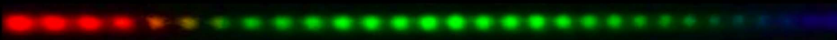

FIG. 7 Experiment 2. Observed fringe pattern for the staircase object. Here, $K=5$. $w \approx 3 \mathrm{~mm}$ and $d=100 \mu \mathrm{m}$.

The intensity distribution of the fringe pattern can be calculated with the theory presented earlier. The linear staircase generates $K$ beamlets with multiple delays: $t_{r}^{(k)}=k \tau_{r}$. We obtain:

$$
S(t, \alpha)=\sum_{k=0}^{K-1} \sum_{m=0}^{M-1} S_{0}\left[t-(m+k M) t+k t_{r}\right]
$$

The intensity of the modulation varies with:

$$
|\tilde{F}(v, \alpha)|^{2}=\left|\sum_{k=0}^{K-1} \exp \left[2 \pi i k v\left(M \tau-t_{r}\right)\right]\right|^{2}=\frac{\sin ^{2}(\pi K \phi)}{\sin ^{2}(\pi \phi)}
$$

For the linear staircase, we thus obtain a modulation of the Talbot bands as it is typical for a multiple beam interferometer with $K$ beams. Thus the peaks appear sharper than for the classical Talbot band experiment (see Figure 7).

In experiment 3, a simple binary phase grating is used (Figure 8 ). The period $2 w$ of this element is rather coarse, so that diffraction can be neglected. Spatially, the structure subdivides the incoming beam into $K$ beamlets as in experiment 2 with a similar reduction of the step height. Temporally, however, only a single time delay occurs as in experiment 1 . Hence that pattern is also sinusoidal. However, the smaller step height results in a shorter time delay than in experiment 1 . Therefore in the $v$-domain a fringe period is observed whose period is increased (Figure 9). In the angular domain, the period is $p_{T}^{\prime}=K \lambda / W$. It should be noted, that in the case of an object with lateral symmetry, one observes a fringe pattern also in the -1st order since pairs of pulses also form for direction $-\alpha$.

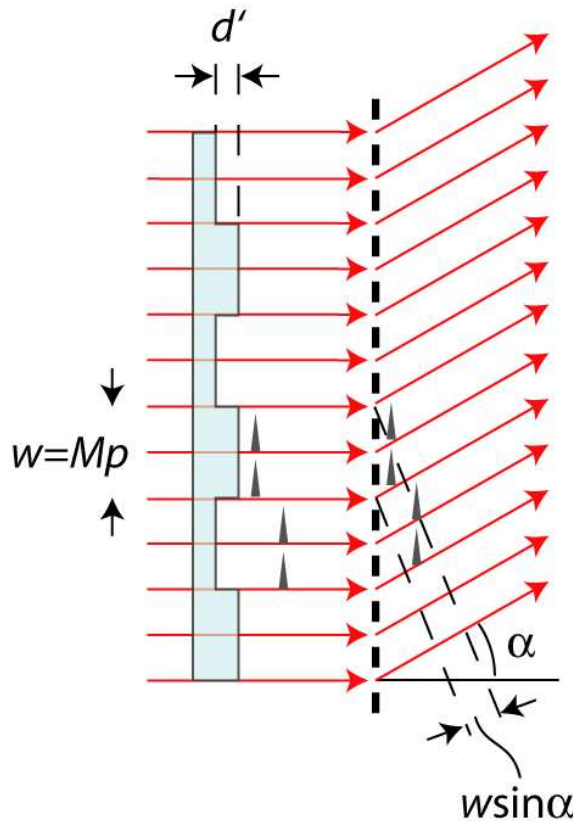

FIG. 8 Experiment 3 with coarse binary grating as object. Here, $w=2000 \mu \mathrm{m}$ and $d \approx 20 \mu \mathrm{m}$.

FIG. 9 Experiment 3. Fringe pattern with sinusoidal modulation.

As a final remark we would like to add: we have so far only considered objects with a piece-wise constant phase structure. However, this is not a necessary restriction. Special combinations of refractive and diffractive elements might also be useful, for example, a lens combined with a Fresnel zone plate.

\section{C ONCLUSION AND OUTLOOK}

So far, the Talbot band experiment has been mostly considered as a curiosity and as an example of the role of temporal aspects in optical interferometry. Here, we have considered a different point of view: the Talbot band experiment suggests that a combination of two different kinds of dispersion, diffractive and refractive, yields surprising results. That may be inspiring for the so-called "dispersion management" which is an important issue for temporal signal processing at ultra high speed. Yet more general effects might emerge if also a third tool, reflective dispersion, is used in a mixed fashion. That idea becomes clear by considering in Figure10, a reflective implementation of the classical Talbot band experiment of Fig. 1. Here, a micro machined mirror causes the delay between the left and the right half of the input aperture. 


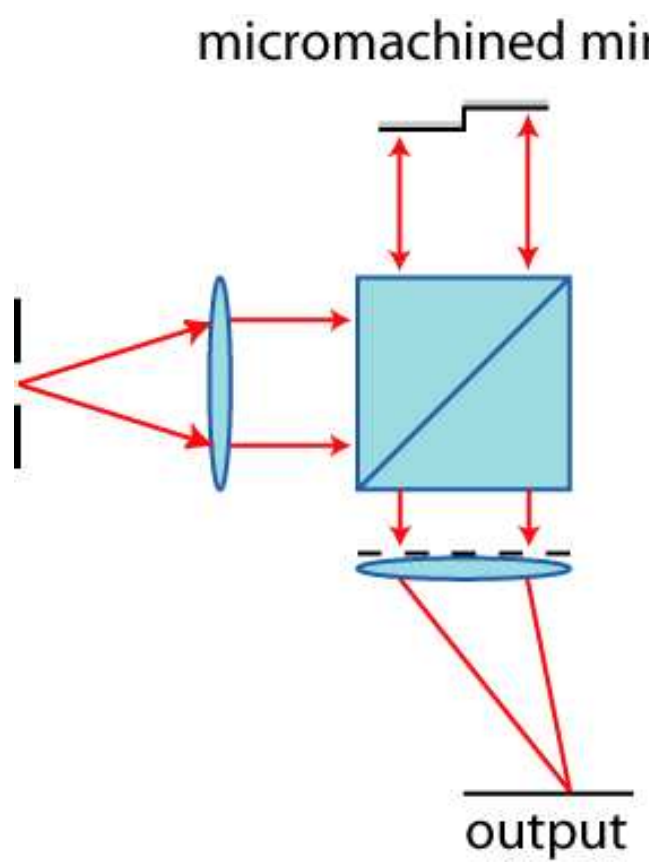

FIG. 10 Reflective optical setup for realisation of Talbot bands.

The use of micromachined optical elements with depths ranging between 10 and $1000 \mu \mathrm{m}$ allows one to implement optical delays from the fs- up to the ps-range. This may be used to implement an optical pulse shaper based on the Talbot band experiment beside well-known setups such as the Treacy interferometer [8] and the widely used far-field grating interferometer, GI [9]. Far-field GI and the Talbot band setup are shown in a combined drawing in Figure 11. In the GI, a first grating, $\mathrm{G}_{1}$, forms the spectrum in the filter plane $\mathrm{F}$ (denoted by the coordinate $v$ ). A mask is placed in this plane as a spectral filter. A second grating, $G_{2}$, recombines the different wavelength components. In the Talbot experiment, the phase element is positioned in the Fourier-conjugate plane near the first grating. In comparison one may say that in the GI-setup filtering is performed in the frequency domain, in the Talbot band setup we influence directly the temporal shape of the signal. A pointlike filter, for example, in the $v$-domain corresponds to a prismatic structure (the linear staircase) in the temporal domain.

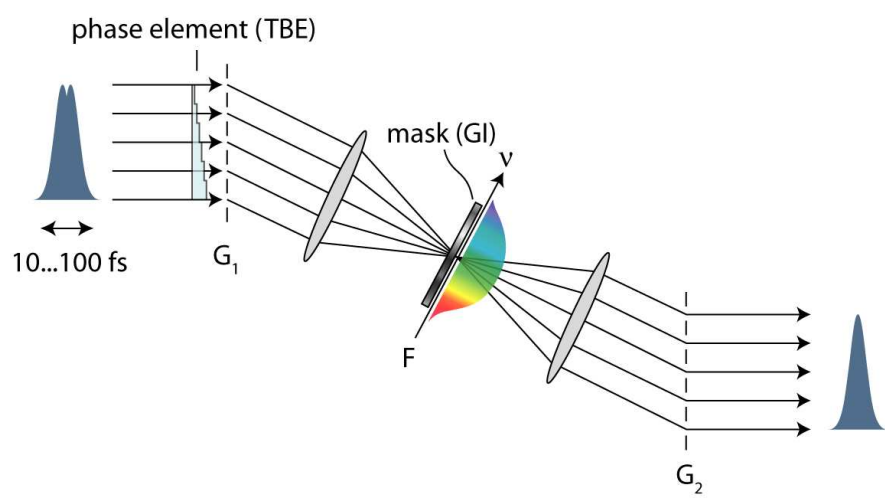

FIG. 11 Optical filtering of fs-pulses. In the conventional grating interferometer setup according to Ref. [9], a mask is used in the filter plane F. Alternatively, a Talbot band setup with a micro machined phase element before grating $G_{1}$ can be used.

\section{References}

[1] H. F. Talbot, "Facts relating to optical sciences," Phil. Mag. 9 401407 (1836).

[2] H. F. Talbot, "An experiment on the interference of light," Phil. Mag. 10364 (1837).

[3] A. W. Lohmann, "Talbot bands" in: Optical information processing, part II ( Uttenreuth 1979 and Erlangen 2006 ch. 6).

[4] M. Parker Givens, "Talbot's bands," Am. J. Phys. 61 601-605 (1993).

[5] Z. Benkö, M. Hilbert, Z. Bor, “New considerations on Talbot's bands," Am. J. Phys. 68 513-520 (2000).

[6] W. H. Knox, R. L. Fork, M. C. Downer, R. H. Stolen, C. V. Shank, J. A. Valdmanis, "Optical pulse compression to 8 fs at a $5-\mathrm{kHz}$ repetition rate", Appl. Phys. Lett. 46 1120-1121 (1985).

[7] E. N. Leith, G. J. Swanson, "Achromatic interferometers for white light optical processing and holography," Appl. Opt. 19 638-644 (1980).

[8] E. B. Treacy, "Optical pulse compression with diffraction gratings," IEEE J. Quantum Electron. QE-5 454-458 (1969).

[9] C. Froehly, B. Colombeau, M. Vampouille, "Shaping and analysis of picosecond light pulses," in: Progress in Optics, E. Wolf, ed. North-Holland, Amsterdam, 20 63-153 (1983). 